\title{
Time-Use and Mental Health in Older Adults: A Scoping Review
}

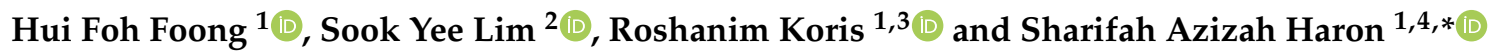 \\ 1 Malaysian Research Institute on Ageing (MyAgeingTM), Universiti Putra Malaysia, \\ Serdang 43400, Selangor, Malaysia; huifoh@upm.edu.my (H.F.F.); \\ roshanim@upm.edu.my or roshanim@umt.edu.my (R.K.) \\ 2 Department of Dietetics, Faculty of Medicine and Health Sciences, Universiti Putra Malaysia, \\ Serdang 43400, Selangor, Malaysia; 1.sookyee@yahoo.com \\ 3 Faculty of Business, Economics and Social Development, Universiti Malaysia Terengganu, \\ Kuala Nerus 21030, Terengganu, Malaysia \\ 4 Department of Resource Management and Consumer Studies, Faculty of Human Ecology, \\ Universiti Putra Malaysia, Serdang 43400, Selangor, Malaysia \\ * Correspondence: sh.azizah@upm.edu.my; Tel.: +60-3-9769-7108
}

Citation: Foong, H.F.; Lim, S.Y.; Koris, R.; Haron, S.A. Time-Use and Mental Health in Older Adults: A Scoping Review. Int. J. Environ. Res. Public Health 2021, 18, 4459. https:// doi.org/10.3390/ijerph18094459

Academic Editor: Paul Tchounwou

Received: 28 January 2021

Accepted: 22 February 2021

Published: 22 April 2021

Publisher's Note: MDPI stays neutral with regard to jurisdictional claims in published maps and institutional affiliations.

Copyright: (c) 2021 by the authors. Licensee MDPI, Basel, Switzerland. This article is an open access article distributed under the terms and conditions of the Creative Commons Attribution (CC BY) license (https:/ / creativecommons.org/licenses/by/ $4.0 /)$.

\begin{abstract}
Time-use of older adults can be different than in earlier life, especially during the transition from pre- to post-retirement or after experiencing major life events, and the changes could affect their mental health. However, the extent and nature of such research in gerontology have not been examined to date. Therefore, this scoping review sought to map the literature on time-use and mental health in the older population to examine the extent and nature of those research activities. A scoping review was conducted using four databases-PubMed, Scopus, CINAHL, and EMBASE according to PRISMA guidelines. Data were extracted using a pretested tool to develop a descriptive analysis and thematic summary. A total of 11 articles met the eligibility criteria. Seven out of 11 studies involved cross-sectional design, while the remainder were longitudinal studies. The longitudinal studies mainly were secondary data analysis. Time-use data were mainly collected using daily diaries, and the most common mental health outcome included was depression. Only two studies did not evaluate the direct relationship between time-use and mental health. Our review has revealed studies evaluating time-use and mental health in older adults. Limitations of review and recommendations for future studies are discussed.
\end{abstract}

Keywords: time use; daily diary; mental health; depression; happiness

\section{Introduction}

The time-use study aims to examine how a person occupies time. Although identification of time-use in older adults has been labeled as an important direction, literature on how older adults spend their time still lacks, especially in Asian countries [1]. Most of the existing empirical studies are from developed Western countries. The time-use research in older adults started as early as 1997 when McLennan reported that older people aged 65 and above in Australia spent most of their time in personal care, domestic, and leisure activities [2]. Gauthier and Smeeding concluded that older adults from nine countries in Europe and North America spent more time watching television, reading, and listening to the radio after they stopped working in paid jobs [3]. In Asia, according to Punyakaew et al., older adults in a Thailand suburban community spent around $8.6 \mathrm{~h}$ on rest and sleep, followed by $6.3 \mathrm{~h}$ on leisure activities and $4.9 \mathrm{~h}$ on work [4].

Observation, experience sampling, and time diaries are three possible methods to collect time-use data, and each has strengths and drawbacks [5]. Observational methods are useful in collecting detailed information about time-use in a particular environment and the number of times a behavior has occurred in a small sample study; however, it requires 
human resources for the observation and is potentially intrusive [1]. Researchers may consider using experience sampling if they intend to minimize the typical time-use data and to know more about time-use in randomly chosen portions of time. The strength of this method is it does not require memory as this is not a recall method. However, this method does not tell patterns of activity and the frequency of activities [6]. Time diaries are the best and most common approach to collect time-use data [7]. Time diaries collected detailed information on the types of activity, the duration of activity and activity patterns with time intervals ranging from $5 \mathrm{~min}$ to an hour interval; however, this method has been criticized for bias to the data due to the retrospective recall [1]. Therefore, researchers suggested using larger time intervals $(30 \mathrm{~min}$ ) to improve recall accuracy [7]. Productive time-use means active lifestyle and involvement in various activities, such as physical exercise, hobbies, social, and leisure activities. Past studies have shown that practicing an active lifestyle by participating in physical activity and social activities are associated with better cognitive function $[8,9]$, higher wellbeing $[10,11]$, and lower depressive symptoms $[12,13]$ in older adults.

Population aging across the world causes implications not only for the disease burden but also for the social and healthcare system [14]. For instance, the number of older people with mental disorders is expected to double by 2030, and statistics have shown that approximately $15 \%$ of older adults aged 60 and above experience mental disorders, such as depression, anxiety, isolation, or dementia [15]. Depression appears as one of the most common mental disorders in older people [16]. Previous studies have shown that good mental health in old age promotes not only quality of life [17] but also longevity [18]. There is compelling evidence that being active in old age is associated with good mental health. For example, several studies have concluded that physical activity reduces the risk of depression in older adults $[19,20]$. Moreover, physical activity is also associated with better optimism, life satisfaction, positive affect, and psychological wellbeing in older adults living with loneliness [21].

Activity patterns of older adults can be different than in earlier life, and the changes in time-use and activity pattern on mental health are crucial as good mental health is an important element in successful aging [22]. Moreover, activity engagement and active lifestyle remain central in several models of healthy aging, such as the successful aging model [23-25] and the productive engagement framework [26,27]. Since no reviews have examined this topic, a clear need exists to comprehensively review the studies of time-use and mental health in older adults. This scoping review aims to map the literature on time-use and mental health in the older population to examine the extent and nature of those research activities.

\section{Materials and Methods}

\subsection{Research Question}

The development of this scoping review followed the guidelines by Arksey and O'Malley [28], which started from developing a research question. As suggested by Richardson and colleagues, [29] the research question was determined according to the population, intervention, controls, and outcomes. This scoping review aims to resolve the following research question "What is the extent and nature of research activities on time-use and mental health among older adults?"

\subsection{Search Strategy}

Two investigators independently searched PubMed, Scopus, CINAHL, and EMBASE databases for potential studies published in journals from inception to November 2020. There was no limit on the years of publication, as long as it was published before or on 30 November 2020. The search was restricted to studies focusing on time-use and mental health in older adults. Table 1 presents the search terms used in the present review. 
Table 1. Search terms.

\begin{tabular}{ccc}
\hline & Variables & Target Group \\
\hline activity pattern OR time OR & mental health OR psychological & \\
diary OR time-use OR time & health OR psychological distress & elderly OR aging OR \\
budget OR yesterday diary OR & OR psychological OR & geriatric OR over-65 \\
time studies OR time utilization & AND & OR older \\
$\begin{array}{c}\text { OR daily activities OR time } \\
\text { allocation }\end{array}$ & psychosocial OR mental OR life & satisfaction \\
\hline
\end{tabular}

\subsection{Eligibility Criteria}

Table 2 shows the domain, inclusion and exclusion criteria, and rationale of the criteria. The inclusion and exclusion criteria were:

- Any study from inception until 30 November 2020;

- Studies from any country;

- Studies only in English;

- Studies only published in peer-review journals;

- Quantitative study with either experimental, longitudinal, cross-sectional, or correlational designs so that the relationship between time-use and mental health could be evaluated if the study did the analysis;

- Studies that involved human time-use and mental health involving older adults. Studies that involved both older adults and non-older adults were permitted with the condition that the involvement of non-older adults was so that the findings on older adults could be compared with non-older adults;

- Studies that collected time-use on a broad range of daily activities, but not discrete activity in isolation. Studies that collected activity engagement using a frequency scale or with "yes/no" responses were excluded as they cannot provide information on activity duration.

Table 2. Inclusion and exclusion criteria.

\begin{tabular}{|c|c|c|c|}
\hline Domain & Inclusion Criteria & Exclusion Criteria & Rationale \\
\hline 1. Publication year & $\begin{array}{l}\text { Studies from inception until } \\
30 \text { November } 2020\end{array}$ & $\begin{array}{l}\text { Studies published after } \\
30 \text { November } 2020\end{array}$ & $\begin{array}{l}\text { We did a preliminary search } \\
\text { using the search terms at } \\
\text { Google Scholar and found } \\
\text { that the number of papers } \\
\text { available was not } \\
\text { overwhelmed }\end{array}$ \\
\hline 2. Publication type & $\begin{array}{l}\text { Studies published in } \\
\text { peer-reviewed journals only }\end{array}$ & $\begin{array}{l}\text { Studies, reports, or other } \\
\text { materials not published in } \\
\text { peer-reviewed journals }\end{array}$ & $\begin{array}{l}\text { To ensure the academic rigor } \\
\text { and quality of the studies }\end{array}$ \\
\hline 3. Research design & $\begin{array}{l}\text { Quantitative studies involved } \\
\text { experimental, longitudinal, and } \\
\text { correlational study design }\end{array}$ & $\begin{array}{c}\text { Qualitative study or any study } \\
\text { not involved quantitative } \\
\text { research design }\end{array}$ & $\begin{array}{l}\text { To evaluate the relationship } \\
\text { between time-use and mental } \\
\text { health if the study did } \\
\text { examine for it }\end{array}$ \\
\hline 4. Study scope/variables & $\begin{array}{l}\text { Studies that involved collecting } \\
\text { human time-use and mental } \\
\text { health data }\end{array}$ & $\begin{array}{l}\text { Studies that collected discrete } \\
\text { activity in isolation } \\
\text { Studies that collected activity } \\
\text { engagement using frequency } \\
\text { scale or with "yes/no" } \\
\text { response }\end{array}$ & $\begin{array}{l}\text { To ensure that the review } \\
\text { question is addressed } \\
\text { Frequency scale or "yes/no" } \\
\text { response cannot provide } \\
\text { information on activity } \\
\text { duration }\end{array}$ \\
\hline
\end{tabular}


Table 2. Cont.

\begin{tabular}{|c|c|c|c|}
\hline Domain & Inclusion Criteria & Exclusion Criteria & Rationale \\
\hline 5. Target group & $\begin{array}{l}\text { Older adults with no specific age } \\
\text { Studies involved both older } \\
\text { adults, and non-older adults were } \\
\text { permitted with the condition that } \\
\text { the involvement of non-older } \\
\text { adults was to so that the findings } \\
\text { on older adults could be } \\
\text { compared with non-older adults }\end{array}$ & $\begin{array}{l}\text { Studies that did not involve } \\
\text { older adults at all }\end{array}$ & $\begin{array}{c}\text { To ensure that the review } \\
\text { question is addressed }\end{array}$ \\
\hline 6. Location & Any country & Not applicable & $\begin{array}{l}\text { Analyzing studies from } \\
\text { Western and non-Western } \\
\text { countries could help } \\
\text { researchers in gaining deeper } \\
\text { insight into the cultural } \\
\text { differences in older adults' } \\
\text { time-use and mental health }\end{array}$ \\
\hline
\end{tabular}

\subsection{Screening}

First, two investigators independently imported the potential articles from the databases into Endnote Program X5 Version. Any duplicate publications were removed manually and by Endnote Program X5. Second, the titles and abstracts were screened independently by them for suitability based on the search strategies mentioned above. Then, full-text articles were independently assessed based on the inclusion and exclusion criteria. Lastly, they performed a manual hand-search for the potential articles from the citation of the included articles. All included articles were agreed upon after discussing them with the third investigator.

\subsection{Data Extraction}

The following data were extracted by two investigators independently using a standardized, comprehensive, and piloted data extraction template- the last name of the first author and year of publication, country, study objective, study design, sample characteristics, the instrument used to measure time-use, type of mental health measured, the instrument to assess mental health, main statistical analysis, and study findings. The disagreement was resolved after consulting the third investigator. Quality appraisal of each study was not performed as this is not a compulsory procedure in scoping review [30].

\subsection{Data Analysis}

All authors read, understood and synthesized the findings. Several discussions were conducted among the study group to categorize findings into the following themes [30]; "demographic, time-use, and mental health," "changes in time-use and mental health," work status, types of activities, and mental health," and "activity profiles and mental health."

\section{Results}

\subsection{Study Characteristics}

Figure 1 illustrates the results from the search and study selection processes. The systematic search of electronic databases yielded 2167 potential studies with 11 articles published between 1995 and 2019 that met the inclusion criteria for this review (see Table 3). In general, the number of studies did not increase by each year except for a peak of three studies in 2018. The regions represented in the review showed that authors from the United States contributed the most studies $(n=6,54.5 \%)$, followed by Australia $(n=3,27.3 \%)$, Netherlands $(n=1,9.1 \%)$, and one study $(n=1,9.1 \%)$ had combined data from Italy, Spain, United Kingdom, France, and The Netherlands. All studies were from developed European countries and none from Asian countries. 


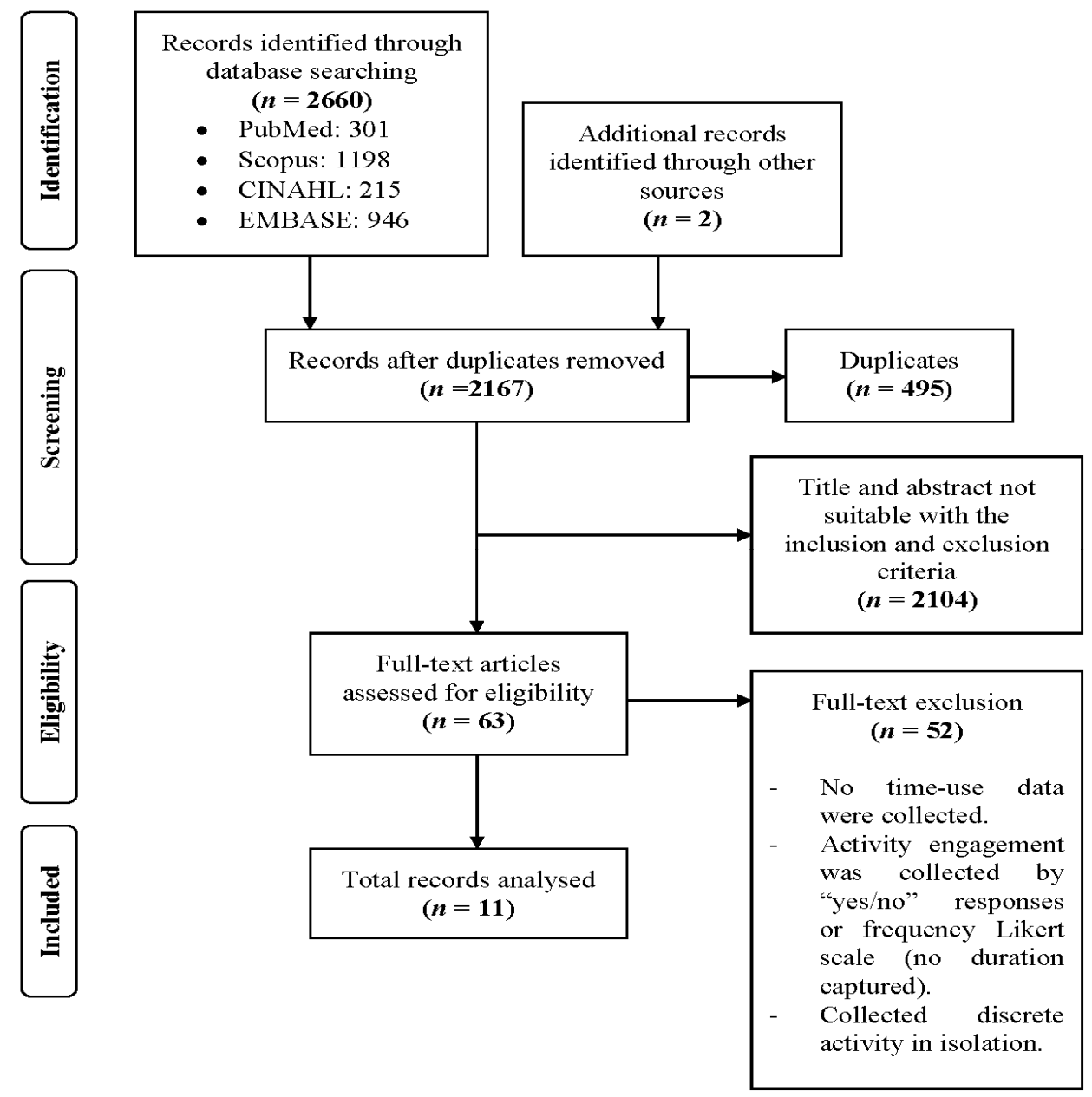

Figure 1. Scoping review flow chart.

Table 3. Number of studies published by year.

\begin{tabular}{lc}
\hline Year & Number of Studies \\
\hline 1995 & 1 \\
2003 & 1 \\
2007 & 1 \\
2011 & 1 \\
2012 & 1 \\
2013 & 1 \\
2014 & 1 \\
2018 & 3 \\
2019 & 1 \\
\hline
\end{tabular}

The summary of all the included study is presented in Table 4 . Six studies (54.5\%) involved secondary data analysis, and the other five studies (45.5\%) involved primary data analysis. Two studies (18.2\%) analyzed data from the National Study of Daily Experiences in the United States. The other two studies $(18.2 \%)$ drew the health and retirement study data linked with consumption and activity mail survey from the United States. One study (9.1\%) analyzed data from the multinational time-use study, which combined data from five different countries; Italy, Spain, United Kingdom, France, and the Netherlands. Another study $(9.1 \%)$ obtained data from a driving cessation study in Australia. In terms of study design, most of the studies $(n=7,63.6 \%)$ were cross-sectional. Four studies $(36.4 \%)$ had a longitudinal study design. 
Table 4. Summary of studies.

\begin{tabular}{|c|c|c|c|c|c|c|c|c|c|}
\hline Author, Y & Country & Study Objective & Study Design & $\begin{array}{c}\text { Sample } \\
\text { Characteristics }\end{array}$ & $\begin{array}{l}\text { Instrument Used to } \\
\text { Measure Time Use }\end{array}$ & $\begin{array}{l}\text { Type of Mental } \\
\text { Health Measured }\end{array}$ & $\begin{array}{c}\text { Instrument to } \\
\text { Assess Mental } \\
\text { Health }\end{array}$ & $\begin{array}{l}\text { Main Statistical } \\
\text { Analysis }\end{array}$ & Study Findings \\
\hline $\begin{array}{l}\text { 1. (Prigerson et al. } \\
\text { 1995) [31] }\end{array}$ & $\begin{array}{l}\text { United } \\
\text { States }\end{array}$ & $\begin{array}{c}\text { To determine if } \\
\text { high regularity in } \\
\text { the timing of daily } \\
\text { activities was } \\
\text { protective against } \\
\text { depressive } \\
\text { symptoms among } \\
\text { older adults soon } \\
\text { after spousal } \\
\text { death }\end{array}$ & $\begin{array}{l}\text { Cohort study The } \\
\text { regularity of daily } \\
\text { activities was } \\
\text { assessed at } 3 \\
\text { months post-loss; } \\
\text { depressive } \\
\text { symptoms were } \\
\text { measured at } 3,12 \text {, } \\
\text { and } 24 \text { months } \\
\text { post-loss }\end{array}$ & $\begin{array}{c}47 \text { older adults } \\
\text { aged between } 60 \\
\text { and above who } \\
\text { just lost their } \\
\text { spouse; } \\
\text { The distribution of } \\
\text { the sample by sex } \\
\text { and description of } \\
\text { sample's age was } \\
\text { not provided }\end{array}$ & $\begin{array}{l}\text { Time use measured by } \\
\text { using the social } \\
\text { rhythm metric (SRM); } \\
\text { The instrument is } \\
\text { diary-like in form, } \\
\text { requiring the subject } \\
\text { to provide daily } \\
\text { details of the time of } \\
\text { day at which each } \\
\text { event occurred; } \\
\text { Data for each week } \\
\text { were then analyzed to } \\
\text { come out with an SRM } \\
\text { score; } \\
\text { The score lies on a } \\
\text { continuum between } 0 \\
\text { and } 7 \text {, with } 0 \\
\text { representing greatest } \\
\text { irregularity and } 7 \\
\text { greatest regularity }\end{array}$ & $\begin{array}{l}\text { Depressive } \\
\text { symptoms }\end{array}$ & $\begin{array}{l}\text { 17-Item Hamilton } \\
\text { rating scale for } \\
\text { depression }\end{array}$ & $\begin{array}{l}\text { Multiple } \\
\text { regression }\end{array}$ & $\begin{array}{l}\text { Baseline SRM was not } \\
\text { associated with } \\
\text { severity of depression } \\
\text { at 12- or 24-month } \\
\text { post-loss; } \\
\text { For those with activity } \\
\text { level index (ALI) } \\
\text { scores of } 80 \text { or above, } \\
\text { lifestyle regularity was } \\
\text { negatively associated } \\
\text { with depressive } \\
\text { symptoms at } \\
\text { 12-month post-loss; } \\
\text { In subjects with ALI } \\
\text { scores of } 90 \text { or above, } \\
\text { baseline lifestyle } \\
\text { regularity was } \\
\text { associated with lower } \\
\text { levels of depressive } \\
\text { symptoms at } \\
\text { 24-month post-loss }\end{array}$ \\
\hline $\begin{array}{l}\text { 2. (Jennings and } \\
\text { Darwin, 2003) [32] }\end{array}$ & $\begin{array}{l}\text { United } \\
\text { States }\end{array}$ & $\begin{array}{l}\text { To evaluate the } \\
\text { relationship } \\
\text { between daily } \\
\text { activities and } \\
\text { memory } \\
\text { performance in } \\
\text { older adults }\end{array}$ & $\begin{array}{l}\text { Cross-sectional } \\
\text { study }\end{array}$ & $\begin{array}{c}\text { Group 1:29 older } \\
\text { adults, aged from } \\
69 \text { to } 93 \text { y (mean } \\
\text { age = } 78.1 \text { ); } \\
\text { Group 2:30 } \\
\text { undergraduate } \\
\text { students, aged } 18 \\
\text { to } 20 \text { y (mean = } \\
18.70) ; \\
\text { Distribution of } \\
\text { respondents by } \\
\text { sex was not } \\
\text { provided }\end{array}$ & $\begin{array}{c}\text { Participants were } \\
\text { asked to indicate how } \\
\text { many hours per week } \\
\text { and how many weeks } \\
\text { per month they } \\
\text { participated in a } \\
\text { variety of physical } \\
\text { activities, mental } \\
\text { activities, and social } \\
\text { activities }\end{array}$ & $\begin{array}{c}\text { Memory } \\
\text { performance }\end{array}$ & $\begin{array}{l}\text { California verbal } \\
\text { learning test }\end{array}$ & $\begin{array}{l}\text { Independent } \\
\text { sample } t \text {-test }\end{array}$ & $\begin{array}{l}\text { Time-use in physical } \\
\text { activity was positively } \\
\text { associated with } \\
\text { memory performance; } \\
\text { No association was } \\
\text { noted between } \\
\text { time-use in social and } \\
\text { mental activity with } \\
\text { memory performance }\end{array}$ \\
\hline
\end{tabular}


Table 4. Cont.

\begin{tabular}{|c|c|c|c|c|c|c|c|c|c|}
\hline Author, Y & Country & Study Objective & Study Design & $\begin{array}{c}\text { Sample } \\
\text { Characteristics }\end{array}$ & $\begin{array}{l}\text { Instrument Used to } \\
\text { Measure Time Use }\end{array}$ & $\begin{array}{l}\text { Type of Mental } \\
\text { Health Measured }\end{array}$ & $\begin{array}{c}\text { Instrument to } \\
\text { Assess Mental } \\
\text { Health }\end{array}$ & $\begin{array}{l}\text { Main Statistical } \\
\text { Analysis }\end{array}$ & Study Findings \\
\hline $\begin{array}{l}\text { 3. (Mckenna, } \\
\text { Broome, and } \\
\text { Liddle, 2007) [5] }\end{array}$ & Australia & $\begin{array}{l}\text { To describe the } \\
\text { time-use profile } \\
\text { and role } \\
\text { participation in } \\
\text { community- } \\
\text { dwelling older } \\
\text { adults; } \\
\text { to analyze if } \\
\text { time-use and role } \\
\text { participation } \\
\text { changed with } \\
\text { increasing age, } \\
\text { and to examine } \\
\text { the relationship } \\
\text { between role } \\
\text { participation and } \\
\text { life satisfaction }\end{array}$ & $\begin{array}{l}\text { Cross-sectional } \\
\text { study Secondary } \\
\text { data analysis of a } \\
\text { driving cessation } \\
\text { study }\end{array}$ & $\begin{array}{l}\text { Total sample }=195 \\
\text { community- } \\
\text { dwelling older } \\
\text { adults ( } 81 \text { men } \\
\text { and } 114 \text { women); } \\
\text { Majority of them } \\
\text { were aged from } \\
\text { 65-74 y old } \\
(n=94), \text { followed } \\
\text { by 75-84 y }(n=79), \\
\text { and } 85+\text { y }(n=22)\end{array}$ & $\begin{array}{l}\text { Activity configuration } \\
\text { was used to record } \\
\text { time-use } \\
\text { retrospectively; } \\
\text { Participants were } \\
\text { asked to recall their } \\
\text { activities from the past } \\
\text { week in half-hour } \\
\text { intervals }\end{array}$ & Life satisfaction & $\begin{array}{l}\text { Life satisfaction } \\
\text { index-Z }\end{array}$ & $\begin{array}{c}\text { ANOVA, } \\
\text { chi-squared } \\
\text { analysis, } \\
\text { independent } \\
\text { sample } t \text {-test, } \\
\text { linear regression }\end{array}$ & $\begin{array}{l}\text { Participants spent } \\
\text { most of the time on } \\
\text { sleep, followed by } \\
\text { solitary activities and } \\
\text { social leisure; } \\
\text { The most common } \\
\text { roles were friend, } \\
\text { family member, and } \\
\text { home maintainer; } \\
\text { Participants aged } 75 \text { y } \\
\text { and older spent } \\
\text { significantly more } \\
\text { time on solitary leisure } \\
\text { and less time on paid } \\
\text { work and transport } \\
\text { compared to those } \\
\text { aged } 65-74 \text { y; } \\
\text { Role maintenance was } \\
\text { significantly related to } \\
\text { greater life satisfaction } \\
\text { in participants aged } \\
75-84 \text { y; } \\
\text { This study did not } \\
\text { examine the } \\
\text { relationship between } \\
\text { time-use and life } \\
\text { satisfaction }\end{array}$ \\
\hline
\end{tabular}


Table 4. Cont.

\begin{tabular}{|c|c|c|c|c|c|c|c|c|c|}
\hline Author, Y & Country & Study Objective & Study Design & $\begin{array}{c}\text { Sample } \\
\text { Characteristics }\end{array}$ & $\begin{array}{l}\text { Instrument Used to } \\
\text { Measure Time Use }\end{array}$ & $\begin{array}{l}\text { Type of Mental } \\
\text { Health Measured }\end{array}$ & $\begin{array}{c}\text { Instrument to } \\
\text { Assess Mental } \\
\text { Health }\end{array}$ & $\begin{array}{c}\text { Main Statistical } \\
\text { Analysis }\end{array}$ & Study Findings \\
\hline $\begin{array}{l}\text { 4. (Hahn, Cichy, } \\
\text { Almeida, and } \\
\text { Haley, 2011) [33] }\end{array}$ & $\begin{array}{l}\text { United } \\
\text { States }\end{array}$ & $\begin{array}{l}\text { To compare daily } \\
\text { time-use and daily } \\
\text { wellbeing in } \\
\text { widowed and } \\
\text { married women }\end{array}$ & $\begin{array}{l}\text { Cross-sectional } \\
\text { study; } \\
\text { Secondary data } \\
\text { analysis; } \\
\text { Data source: } \\
\text { second wave } \\
\text { (2004-2006) of } \\
\text { National study of } \\
\text { daily experiences }\end{array}$ & $\begin{array}{c}75 \text { widowed } \\
\text { women }(\text { mean age } \\
=72.4 \pm 6.67) \text { and } \\
125 \text { married } \\
\text { women }(\text { mean age } \\
=70.0 \pm 5.97)\end{array}$ & $\begin{array}{l}\text { Respondents were } \\
\text { asked about the daily } \\
\text { time spent for the } \\
\text { following activities: } \\
\text { interacting with } \\
\text { children, performing } \\
\text { household chores, } \\
\text { doing work or school } \\
\text { work, relaxing or } \\
\text { doing leisure activities, } \\
\text { watching TV, } \\
\text { volunteering, giving or } \\
\text { receiving unpaid } \\
\text { assistance, giving or } \\
\text { receiving emotional } \\
\text { support, providing } \\
\text { help to someone with } \\
\text { a disability, and } \\
\text { sleeping; } \\
\text { Daily time use was } \\
\text { collected for } 8 \\
\text { consecutive days }\end{array}$ & Daily wellbeing & $\begin{array}{l}\text { Respondents were } \\
\text { asked how often } \\
\text { during the past } \\
\text { day they } \\
\text { experienced } \\
14 \text { different } \\
\text { negative emotions } \\
\text { and } 13 \text { different } \\
\text { positive emotions } \\
\text { with a Likert scale } \\
\text { ranging from } 0 \\
\text { (none of the time) } \\
\text { to } 4 \text { (all of the } \\
\text { time) }\end{array}$ & $\begin{array}{c}\text { ANCOVA, } \\
\text { independent } \\
\text { samples } t \text {-test }\end{array}$ & $\begin{array}{l}\text { No difference in daily } \\
\text { activity time-use for } \\
\text { most of the activities } \\
\text { between married and } \\
\text { widowed women; } \\
\text { Widowed women } \\
\text { spent more time } \\
\text { accompanying their } \\
\text { children and watching } \\
\text { television and spent } \\
\text { less time sleeping than } \\
\text { married women; } \\
\text { No difference was } \\
\text { noted in wellbeing } \\
\text { between widowed and } \\
\text { married older women }\end{array}$ \\
\hline
\end{tabular}


Table 4. Cont.

\begin{tabular}{|c|c|c|c|c|c|c|c|c|c|}
\hline Author, Y & Country & Study Objective & Study Design & $\begin{array}{c}\text { Sample } \\
\text { Characteristics }\end{array}$ & $\begin{array}{l}\text { Instrument Used to } \\
\text { Measure Time Use }\end{array}$ & $\begin{array}{l}\text { Type of Mental } \\
\text { Health Measured }\end{array}$ & $\begin{array}{c}\text { Instrument to } \\
\text { Assess Mental } \\
\text { Health }\end{array}$ & $\begin{array}{l}\text { Main Statistical } \\
\text { Analysis }\end{array}$ & Study Findings \\
\hline $\begin{array}{c}\text { 5. (Liddle, } \\
\text { Gustafsson, } \\
\text { Bartlett, and } \\
\text { Mckenna, 2012) } \\
\text { [34] }\end{array}$ & Australia & $\begin{array}{l}\text { To examine the } \\
\text { impact of driving } \\
\text { status on time use, } \\
\text { role participation } \\
\text { and life } \\
\text { satisfaction in } \\
\text { older adults }\end{array}$ & $\begin{array}{l}\text { Cross-sectional } \\
\text { study }\end{array}$ & $\begin{array}{c}137 \text { current } \\
\text { drivers (mean age } \\
=73.2 \pm 6.1 \text { ), } \\
56 \text { retired drivers } \\
\text { (mean age = } \\
78.7 \pm 6.7 \text { ), and } \\
41 \text { people who } \\
\text { have never driven } \\
\text { (mean age = } \\
76.2 \pm 6.2 \text { ); } \\
\text { The distribution of } \\
\text { the study sample } \\
\text { by sex was not } \\
\text { reported }\end{array}$ & $\begin{array}{l}\text { Time use was } \\
\text { measured using } \\
\text { activity configuration; } \\
\text { Participants were } \\
\text { asked to recall } \\
\text { activities from the past } \\
\text { week in half-hour } \\
\text { intervals, including } \\
\text { the location of the } \\
\text { activity, presence of } \\
\text { other people and } \\
\text { subjective } \\
\text { classification of the } \\
\text { activity }\end{array}$ & Life satisfaction & $\begin{array}{l}\text { Life satisfaction } \\
\quad \text { index } Z\end{array}$ & $\begin{array}{l}\text { ANOVA, } \\
\text { chi-squared, } \\
\text { multiple } \\
\text { regression, logistic } \\
\text { regression }\end{array}$ & $\begin{array}{l}\text { Those who have never } \\
\text { driven spent more } \\
\text { time doing charity } \\
\text { activities than retired } \\
\text { drivers; } \\
\text { Current drivers spent } \\
\text { more time in social } \\
\text { leisure activities than } \\
\text { retired drivers; } \\
\text { However, retired } \\
\text { drivers spent more } \\
\text { time in solitary leisure } \\
\text { than current drivers; } \\
\text { Current drivers spent } \\
\text { more time away from } \\
\text { home than retired } \\
\text { drivers; } \\
\text { Current drivers had } \\
\text { higher life satisfaction } \\
\text { than retired drivers; } \\
\text { No difference in life } \\
\text { satisfaction between } \\
\text { retired drivers and } \\
\text { people who have } \\
\text { never driven; } \\
\text { This study did not } \\
\text { examine the } \\
\text { relationship between } \\
\text { time-use and life } \\
\text { satisfaction }\end{array}$ \\
\hline
\end{tabular}


Table 4. Cont.

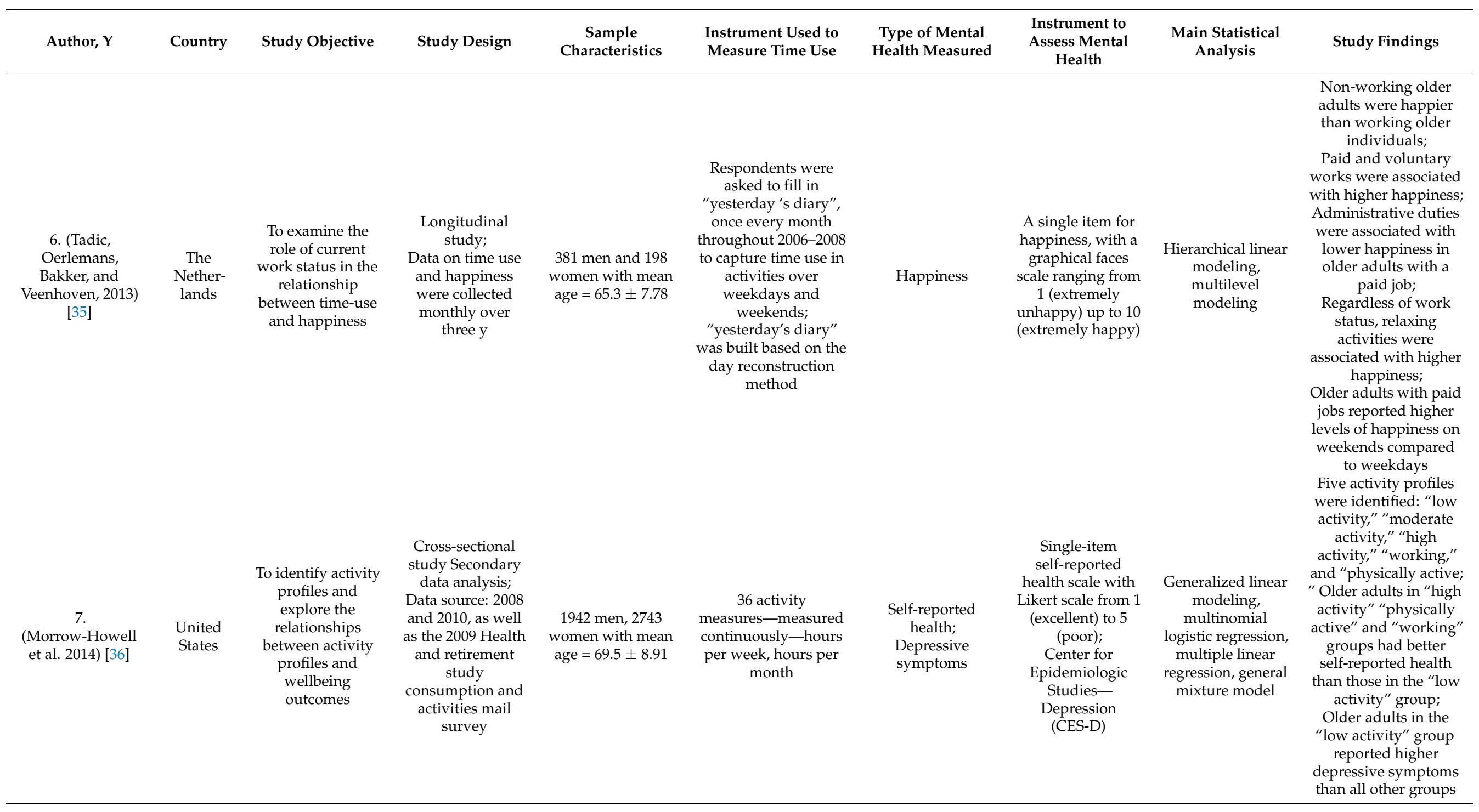


Table 4. Cont.

\begin{tabular}{|c|c|c|c|c|c|c|c|c|c|}
\hline Author, $Y$ & Country & Study Objective & Study Design & $\begin{array}{c}\text { Sample } \\
\text { Characteristics }\end{array}$ & $\begin{array}{l}\text { Instrument Used to } \\
\text { Measure Time Use }\end{array}$ & $\begin{array}{l}\text { Type of Mental } \\
\text { Health Measured }\end{array}$ & $\begin{array}{c}\text { Instrument to } \\
\text { Assess Mental } \\
\text { Health }\end{array}$ & $\begin{array}{l}\text { Main Statistical } \\
\text { Analysis }\end{array}$ & Study Findings \\
\hline $\begin{array}{c}\text { 8. (Adjei, Jonsson, } \\
\text { and Brand, 2018) } \\
\text { [37] }\end{array}$ & $\begin{array}{l}\text { Italy, } \\
\text { Spain, UK, } \\
\text { France } \\
\text { and the } \\
\text { Nether- } \\
\text { lands }\end{array}$ & $\begin{array}{l}\text { To examine the } \\
\text { associations } \\
\text { between } \\
\text { work-related time } \\
\text { use, social time } \\
\text { use and self-rated } \\
\text { health; } \\
\text { To examine if } \\
\text { stress mediated } \\
\text { the relationship } \\
\text { between } \\
\text { work-related time } \\
\text { use activities on } \\
\text { self-reported } \\
\text { health }\end{array}$ & $\begin{array}{l}\text { Cross-sectional } \\
\text { study; } \\
\text { Secondary data } \\
\text { analysis Data } \\
\text { source: } \\
\text { Multinational time } \\
\text { use study (WTUS, } \\
\text { version W53) }\end{array}$ & $\begin{array}{c}11,168 \text { men }(\text { mean } \\
\text { age }=72.4 \pm 5.01) \\
\text { and } 14,295 \text { women } \\
(\text { mean age }= \\
73.1 \pm 5.13)\end{array}$ & $\begin{array}{c}\text { Time use was collected } \\
\text { by self-administered } \\
\text { diary; } \\
\text { Respondents reported } \\
\text { the total time spent on } \\
41 \text { activities over a } 24 \\
\text { h period in 5-, 10- or } \\
\text { 15-min intervals; } \\
\text { Respondents in France, } \\
\text { Italy and Spain } \\
\text { reported the time use } \\
\text { during a randomly } \\
\text { assigned day in a } \\
\text { week; } \\
\text { Respondents from the } \\
\text { UK filled in the diary } \\
\text { for two days (weekday } \\
\text { and weekend); } \\
\text { In the Netherlands, } \\
\text { respondents reported } \\
\text { their time use } \\
\text { activities for seven } \\
\text { consecutive day }\end{array}$ & $\begin{array}{l}\text { Self-rated health; } \\
\text { Stress }\end{array}$ & $\begin{array}{c}\text { One-item } \\
\text { self-reported } \\
\text { health scale with } \\
\text { Likert scale } \\
\text { ranging from } 0 \\
\text { (poor) to } 3 \text { (very } \\
\text { good); } \\
\text { Time pressure was } \\
\text { measured by } \\
\text { asking } \\
\text { respondents, } \\
\text { “Would you say } \\
\text { you always feel } \\
\text { rushed even to do } \\
\text { the things you } \\
\text { must do, only } \\
\text { sometimes feel } \\
\text { rushed, or almost } \\
\text { never feel } \\
\text { rushed?” with } \\
\text { Likert scale } \\
\text { ranging from } 1 \\
\text { (never) to } 3 \\
\text { (always) }\end{array}$ & $\begin{array}{c}\text { Pearson's } \\
\text { correlation, linear } \\
\text { structural model }\end{array}$ & $\begin{array}{l}\text { Housework was } \\
\text { associated with higher } \\
\text { stress in both older } \\
\text { men and women; } \\
\text { Paid work was } \\
\text { associated with lower } \\
\text { stress only in older } \\
\text { men; } \\
\text { Social activities were } \\
\text { associated with better } \\
\text { self-rated health, but } \\
\text { no association was } \\
\text { found with stress; } \\
\text { Stress did not mediate } \\
\text { the association } \\
\text { between housework, } \\
\text { paid work and } \\
\text { self-reported health }\end{array}$ \\
\hline
\end{tabular}


Table 4. Cont.

\begin{tabular}{|c|c|c|c|c|c|c|c|c|c|}
\hline Author, Y & Country & Study Objective & Study Design & $\begin{array}{c}\text { Sample } \\
\text { Characteristics }\end{array}$ & $\begin{array}{l}\text { Instrument Used to } \\
\text { Measure Time Use }\end{array}$ & $\begin{array}{l}\text { Type of Mental } \\
\text { Health Measured }\end{array}$ & $\begin{array}{c}\text { Instrument to } \\
\text { Assess Mental } \\
\text { Health }\end{array}$ & $\begin{array}{l}\text { Main Statistical } \\
\text { Analysis }\end{array}$ & Study Findings \\
\hline $\begin{array}{l}\text { 9. (Lee et al. 2018) } \\
\text { [38] }\end{array}$ & $\begin{array}{l}\text { United } \\
\text { States }\end{array}$ & $\begin{array}{c}\text { To examine the } \\
\text { associations } \\
\text { between activity } \\
\text { diversity and } \\
\text { psychological } \\
\text { wellbeing in } \\
\text { people from } \\
\text { different age } \\
\text { group; } \\
\text { older adults } \\
\text { (60-74 y), } \\
\text { middle-aged } \\
\text { adults (35-59 y), } \\
\text { and younger } \\
\text { individuals } \\
\text { (24-34 y) }\end{array}$ & $\begin{array}{l}10 \text { y longitudinal } \\
\text { study; } \\
\text { Secondary data } \\
\text { analysis; } \\
\text { Data source: } \\
\text { National survey of } \\
\text { daily experiences } \\
\text { (1996-1997 and } \\
\text { 2006-2007) }\end{array}$ & $\begin{array}{c}793 \text { ( } 349 \text { men, } 444 \\
\text { women) } \\
\text { individuals aged } \\
\text { from } 24 \text { to } 74 \text { y at } \\
\text { baseline (mean } \\
\text { age }=46.71 \pm 12.5 \text { ) }\end{array}$ & $\begin{array}{l}\text { 8-day daily diary was } \\
\text { administered to } \\
\text { participants to capture } \\
\text { their daily experiences, } \\
\text { including daily time } \\
\text { use; } \\
\text { Seven activities were } \\
\text { captured: paid work, } \\
\text { with children, doing } \\
\text { chores, on leisure, in } \\
\text { physical activities, on } \\
\text { formal volunteering, } \\
\text { and giving informal } \\
\text { help to people who do } \\
\text { not live with } \\
\text { respondents }\end{array}$ & $\begin{array}{l}\text { Psychological } \\
\text { wellbeing; } \\
\text { Depression } \\
\text { positive and } \\
\text { negative affect }\end{array}$ & $\begin{array}{l}\text { The psychological } \\
\text { wellbeing scale } 7 \\
\text { items of } \\
\text { depressive affect } \\
\text { and } 6 \text { items of } \\
\text { anhedonia; } \\
\text { Positive and } \\
\text { negative affect } \\
\text { scales }\end{array}$ & $\begin{array}{c}\text { Multilevel models, } \\
\text { residualized gain } \\
\text { models, } \\
\text { ANCOVA-type } \\
\text { regression model }\end{array}$ & $\begin{array}{l}\text { Older adults who } \\
\text { engaged in more } \\
\text { diverse activities } \\
\text { reported higher } \\
\text { psychological } \\
\text { wellbeing than older } \\
\text { adults who engaged in } \\
\text { less diverse activities; } \\
\text { Longitudinally, } \\
\text { increased activity } \\
\text { diversity over } 10 \text { y } \\
\text { was associated with } \\
\text { the increases in } \\
\text { positive affect; } \\
\text { Compared with } \\
\text { younger individuals } \\
\text { who increased activity } \\
\text { diversity, older adults } \\
\text { who increased activity } \\
\text { diversity reported } \\
\text { smaller decreases in } \\
\text { psychological } \\
\text { wellbeing, greater } \\
\text { increases in positive } \\
\text { affect, and greater } \\
\text { decreases in negative } \\
\text { affect }\end{array}$ \\
\hline
\end{tabular}


Table 4. Cont.

\begin{tabular}{|c|c|c|c|c|c|c|c|c|c|}
\hline Author, Y & Country & Study Objective & Study Design & $\begin{array}{c}\text { Sample } \\
\text { Characteristics }\end{array}$ & $\begin{array}{l}\text { Instrument Used to } \\
\text { Measure Time Use }\end{array}$ & $\begin{array}{l}\text { Type of Mental } \\
\text { Health Measured }\end{array}$ & $\begin{array}{c}\text { Instrument to } \\
\text { Assess Mental } \\
\text { Health }\end{array}$ & $\begin{array}{c}\text { Main Statistical } \\
\text { Analysis }\end{array}$ & Study Findings \\
\hline $\begin{array}{l}\text { 10. (Olds et al. } \\
\text { 2018) [39] }\end{array}$ & Australia & $\begin{array}{l}\text { To determine the } \\
\text { effects of activity } \\
\text { changes after } \\
\text { retirement on } \\
\text { mental health }\end{array}$ & $\begin{array}{l}\text { Longitudinal } \\
\text { study; } \\
\text { Data on time use, } \\
\text { physical health, } \\
\text { mental health and } \\
\text { sociodemographic } \\
\text { characteristics } \\
\text { were gathered } 6 \\
\text { months before and } \\
\text { at 3-, 6- and } \\
\text { 12-month } \\
\text { post-retirement }\end{array}$ & $\begin{array}{c}54 \text { women and } \\
51 \text { men with } \\
\text { pre-retirement } \\
\text { mean age }= \\
62.3 \pm 4.3 \text { y and } \\
\text { post-retirement } \\
\text { mean age = } \\
63.4 \pm 4.3 \mathrm{y}\end{array}$ & $\begin{array}{l}\text { Time use in activities } \\
\text { was collected by using } \\
\text { multimedia activity } \\
\text { recall for children and } \\
\text { adults (MARCA); } \\
\text { The MARCA was } \\
\text { administered at each } \\
\text { time point on two } \\
\text { occasions, each time } \\
\text { recalling the two } \\
\text { previous days, one } \\
\text { weekday and one day } \\
\text { on the weekend }\end{array}$ & $\begin{array}{c}\text { Depression, } \\
\text { anxiety, and stress } \\
\text { Wellbeing Life } \\
\text { satisfaction } \\
\text { self-esteem }\end{array}$ & $\begin{array}{c}\text { Depression, } \\
\text { anxiety, and stress } \\
\text { scales; } \\
\text { The short } \\
\text { Warwick- } \\
\text { Edinburgh mental } \\
\text { wellbeing scale } \\
\text { Australian unity } \\
\text { personal } \\
\text { wellbeing index } \\
\text { The Rosenberg } \\
\text { self-esteem scale }\end{array}$ & $\begin{array}{l}\text { Regression } \\
\text { analysis, } \\
\text { compositional } \\
\text { isotemporal } \\
\text { substitution } \\
\text { models }\end{array}$ & $\begin{array}{l}\text { Work time flowed } \\
\text { mainly to household } \\
\text { chores, sleep, screen } \\
\text { time and quiet time } \\
\text { after retirement; } \\
\text { Changes in overall } \\
\text { time use were } \\
\text { significantly } \\
\text { associated with lower } \\
\text { depressive symptoms } \\
\text { and stress, as well as } \\
\text { higher self-esteem; } \\
\text { Replacing work time } \\
\text { with physical activity } \\
\text { and sleep was } \\
\text { associated with } \\
\text { improvements in all } \\
\text { measures of mental } \\
\text { health }\end{array}$ \\
\hline
\end{tabular}


Table 4. Cont.

\begin{tabular}{|c|c|c|c|c|c|c|c|c|c|}
\hline Author, Y & Country & Study Objective & Study Design & $\begin{array}{c}\text { Sample } \\
\text { Characteristics }\end{array}$ & $\begin{array}{l}\text { Instrument Used to } \\
\text { Measure Time Use }\end{array}$ & $\begin{array}{l}\text { Type of Mental } \\
\text { Health Measured }\end{array}$ & $\begin{array}{c}\text { Instrument to } \\
\text { Assess Mental } \\
\text { Health }\end{array}$ & $\begin{array}{l}\text { Main Statistical } \\
\text { Analysis }\end{array}$ & Study Findings \\
\hline $\begin{array}{l}\text { 11. (Chen, } \\
\text { Putnam, Lee, and } \\
\text { Morrow-Howell, } \\
\text { 2019) [40] }\end{array}$ & $\begin{array}{l}\text { United } \\
\text { States }\end{array}$ & $\begin{array}{l}\text { To examine the } \\
\text { relationships } \\
\text { between activity, } \\
\text { health, and nature } \\
\text { of engagement in } \\
\text { older adults }\end{array}$ & $\begin{array}{l}\text { Cross-sectional } \\
\text { study Secondary } \\
\text { data analysis Data } \\
\text { source: } 2010 \text { and } \\
2012 \text { Health and } \\
\text { retirement study } \\
\text { linked with } 2011 \\
\text { consumption and } \\
\text { activity mail } \\
\text { survey }\end{array}$ & $\begin{array}{l}3516 \text { women and } \\
2528 \text { men with } \\
\text { mean age } \\
64.4 \pm 10.37 \mathrm{y}\end{array}$ & $\begin{array}{l}33 \text { items that captured } \\
\text { a wide range of } \\
\text { activities that involved } \\
\text { varying degrees of } \\
\text { physical, cognitive, } \\
\text { and social } \\
\text { engagement; } \\
\text { Time use for each } \\
\text { activity was divided } \\
\text { into three levels; } \\
\text { low, medium, and } \\
\text { high }\end{array}$ & $\begin{array}{l}\text { Cognitive function } \\
\text { Depressive } \\
\text { symptoms } \\
\text { Self-rated health }\end{array}$ & $\begin{array}{l}\text { Numbers of } \\
\text { words recalled } \\
\text { immediately and } \\
\text { delayed The } \\
\text { modified version } \\
\text { of the Center for } \\
\text { Epidemiologic } \\
\text { Studies- } \\
\text { Depression scale } \\
\text { Single item of } \\
\text { self-rated health } \\
\text { with Likert scale } 1 \\
=\text { excellent to } \\
5=\text { poor }\end{array}$ & $\begin{array}{l}\text { Latent class } \\
\text { analysis, linear } \\
\text { regression }\end{array}$ & $\begin{array}{l}\text { Five patterns of } \\
\text { activity (high, } \\
\text { medium, low, passive } \\
\text { leisure, working) and } \\
\text { three nature of activity } \\
\text { engagement (full, } \\
\text { partial, minimal) were } \\
\text { identified; } \\
\text { High and working } \\
\text { groups, compared to } \\
\text { the passive leisure } \\
\text { group, showed better } \\
\text { health and cognition } \\
\text { outcomes; } \\
\text { Older adults in the } \\
\text { category of "low" } \\
\text { activity with "full" } \\
\text { engagement reported } \\
\text { higher levels of } \\
\text { self-rated health than } \\
\text { older adults in the } \\
\text { "passive leisure" } \\
\text { group; } \\
\text { Older adults in the } \\
\text { category of "moderate" } \\
\text { activity, "high" } \\
\text { activity, and "working" } \\
\text { group reported lower } \\
\text { levels of depressive } \\
\text { symptoms only when } \\
\text { they fully engage in } \\
\text { the activity }\end{array}$ \\
\hline
\end{tabular}


In terms of the study sample, $45.5 \%$ of the studies involved general communitydwelling older adults. One study $(9.1 \%)$ involved older widowed women, older adults who just lost their spouse, pre-retired older workers, and older drivers. Two studies (18.2\%) involved people from different age groups so that the findings on the older group could be compared with other age groups. For sample size, the studies $(n=3,27.3 \%)$ with secondary data analysis had a bigger sample size (number of participants $>1000$ ). In contrast, five studies (45.5\%) utilized a sample size between 100 and 800, and three studies $(27.3 \%)$ involved a sample size of less than 100. This review also found that two studies (27.3\%) collected time-use and mental health data, but the authors did not examine the relationship between these two variables.

\subsection{Time-Use and Its Measurement}

The measurement of time-use is varied across all studies. Some of the instruments noted from this review were the daily diary [33], multimedia activity recall for children and adults [39], the social rhythm metric [31], activity configuration [5,34], and yesterday's diary [35]. One similarity of all the measurements was that most of them covered time-use recall for both weekdays and weekends. In terms of data collection duration, Hahn and colleagues and Lee and colleagues took daily details for eight consecutive days of the time used to capture the type of activities and their time-use [33,38]. Some researchers chose to measure time-use on a weekly and monthly basis by asking the number of hours spent in a week and month for an activity [36,40]. Moreover, Olds and colleagues took a 2-day time-use (1 weekday and 1 weekend) [39]. Aside from collecting data about time spent on certain activities, researchers also considered other useful information, such as the location of the activity and the presence of other people [34].

Some authors collected time-use data for other purposes of analysis. For example, Prigerson and colleagues collected time-use data and generated the Social Rhythm Score used to investigate the sample's social rhythms and lifestyle regularity [31]. Olds and colleagues examined the time-use in mature workers pre- and post-retirement [39]. They conducted time flow analytics after collecting time-use data and presented the time flow among super domains (e.g., work, transport, social, quiet time, screen, etc.) using a chord diagram [39]. Some studies also categorized selected activities based on domains. For example, Jennings and colleagues clustered a series of activities based on the physical, mental, and social domains [32]. Olds and colleagues listed activities based on transport, social, quiet time, screen, sleep, self-care, chores, physical activity, and work domains [39].

\subsection{Mental Health and Its Measurement}

Depressive symptoms were the most common mental health outcome across all the studies $(n=5,45.5 \%)$ [31,36,38-40]. Examples of instruments used to assess depressive symptoms were the 17-item Hamilton Rating Scale for Depression [31], Center for Epidemiological Studies Depression Scale [36,40], and Depression, Anxiety, and Stress Scale [39]. Lee and colleagues measured depression using seven depression items and six items of anhedonia [38]. Three studies (27.3\%) included self-rated health as the wellbeing outcome, and all of them measured this variable using a single-item self-rated health scale $[36,37,40]$. Three studies $(27.3 \%)$ measured life satisfaction as the mental health outcome $[5,34,39]$. The authors used the Australian Unity Personal Wellbeing Index [39] and the Life Satisfaction Index Z [5,34] to measure life satisfaction. Three studies $(27.3 \%)$ included general psychological wellbeing in their studies $[33,38,39]$. The psychological wellbeing measurements were varied: The short Warwick-Edinburgh mental wellbeing scale [39] and the psychological wellbeing scale [38]. Moreover, Hahn and colleagues measured the sample's daily wellbeing by administering 14 items on different negative affects and 13 items on different positive affects with a 5-point Likert scale [33]. Tadic and colleagues measured happiness in their study using a single item for happiness with a graphical face scale [35]. Two studies (18.2\%) involved cognitive function as the mental health outcome, and both measured cognitive function by administering the verbal memory test [32,40]. 


\subsection{Thematic Summary}

\subsubsection{Demographic, Time-Use and Mental Health}

Adjei and colleagues examined the sex differences in the relationship between workrelated time-use and stress [37]. They found that both older men and women reported higher levels of stress with increasing time-use in managing housework. Moreover, the negative relationship between paid work and stress was only found in older men [37]. Hahn and colleagues examined the differences in daily time-use and wellbeing in widowed and married older women [33]. They did not find any differences in daily time-use for most of the activities. Widowed women spent more time accompanying their children and watching television and less time sleeping than married women. No difference was noted in wellbeing between widowed and married older women [33].

Liddle and colleagues examined the role of driving status in older adults' time-use and life satisfaction [34]. They collected driving status as one of the demographic information aside from age, gender, living situation, and the number of health conditions. They found that older adults who never drove spent more time doing charity work than retired drivers. In addition, current drivers spent more time engaging in social leisure activities than retired drivers [34]. However, retired drivers spent more leisure time in solitary than current drivers, and current drivers spent more time away from home than retired drivers. In terms of mental health, current drivers reported higher life satisfaction than retired drivers, and no difference in life satisfaction was noted between retired drivers and older people who have never driven [34].

\subsubsection{Changes in Time-Use and Mental Health}

Changes in time-use and their association with mental health could be examined using a longitudinal design. Olds and colleagues examined the time-use, depressive symptoms, life satisfaction, wellbeing, and self-esteem of mature workers pre- (6-month before) and post- (3-, 6-, and 12-month after) retirement [39]. Retired individuals spent more time on household chores, sleeping, screen time, and quiet time during retirement. Changes in overall time-use were significantly associated with lower depression and stress, as well as higher self-esteem. Replacing working time with physical activity and sleep was associated with improvements in all measures of mental health [39]. Lee and colleagues investigated the relationships between activity diversity, wellbeing, depression, positive and negative affects by following up on a group of respondents from different age groups for 10 years [38]. The study summarized that increased activity diversity in older adults was associated with greater psychological wellbeing and positive affects and decreased negative affects compared to younger respondents [38].

\subsubsection{Work Status, Types of Activities and Mental Health}

Tadic and colleagues examined the role of work status in the relationship between time-use and happiness in older adults [35]. Overall, non-working older adults were happier than older individuals who were working. Working older adults reported lower levels of happiness with higher time spent in administrative duties. Regardless of work status, engaging in leisure activities was associated with higher levels of happiness. In addition, working older adults reported higher levels of happiness on weekends compared to weekdays [35]. Jennings and colleagues examined the relationship between time-use in different activities (physical, mental, and social) and memory performance [32]. Results showed that physical activity was positively associated with memory performance but not social and mental activities [32].

\subsubsection{Activity Profiles and Mental Health}

Chen and colleagues identified activity patterns and the natures of engagement of older participants by using the time-use data and correlated the activity profiles with cognitive function, depressive symptoms, and self-rated health [40]. They identified five patterns of activity-"high", "moderate", "low", "passive leisure", and "working," as 
well as three natures of engagement- "full", "partial", and "minimal" engagement. Older adults who were in the "high" and "working" group reported better self-rated health, cognitive function, and lower depressive symptoms than those of the "passive leisure" group. Older adults in the category of "low" activity with "full" engagement reported higher levels of self-rated health than older adults in the "passive leisure" group. Older adults in the category of "moderate" activity, "high" activity, and "working" group reported lower levels of depressive symptoms only when they are fully engaged in an activity [40].

Morrow and colleagues identified the sample's activity profiles using time-use data and explored the relationships between activity profiles, self-reported health, and depressive symptoms [36]. They categorized respondents based on five activity profiles—“low activity", "moderate activity", "high activity", "working", and "physically active". Findings showed that older adults in "high activity", "physically active", and "working" groups reported better self-reported health than those in the "low activity" group. Moreover, respondents in the "low activity" group reported higher depressive symptoms than all other groups [36]. Prigerson and colleagues examined if lifestyle regularity was associated with lower levels of depressive symptoms in older adults who just lost their spouse [31] and found that lifestyle regularity was associated with lower depressive symptoms in subjects with Activity Level Index higher than 80 at 12- and 24-months post-loss [31].

\section{Discussion}

\subsection{More Evidence on Time-Use and Mental Health in Older Adults Are Required}

The study of time-use and mental health in older adults still lacks in gerontology as the number of publications remained stable and did not increase over the years. This review found only 11 studies can be included as ten papers have been excluded after the full article screening. Those excluded studies only traced activity involvement by asking the respondents to answer either "yes" or "no" or rate their frequency of participation based on a Likert-scale. No time-use data for each activity was collected. We decided to exclude them as they cannot capture the duration of activities. Therefore, the activities that older adults spent on the most and least cannot be determined. Our decision is supported by Bauman and colleagues when they argued that information of daily activities, such as the order, duration, and characteristics, is essential in the time-use study as that information is useful to determine potential health outcomes and consequences [41]. The findings from this review suggest that gerontologists can work more on the research of older adults time-use and its relation to mental health.

All the studies included were from Western and developed countries. None of the studies were from the Asian perspective. It reflects the Western perspective that dominates the study of time-use and mental health in older adults. Limited studies from Asian countries are a problem because perceptions and meaning of time and mental health may differ across cultures and countries [3,42]. For example, older adults spending more time providing care to grandchildren is more virtually universal in Asia than in Western Europe [43]. Moreover, Asian countries like Singapore, Japan, and South Korea exhibit many older adults who continue to work although reaching retirement age due to rapid population aging. Therefore, they still allocate more time to work compared to older adults in other countries. Consequently, the relationship between different time-use and mental health would differ across cultures and countries [44,45]. The difference in the relationship between time-use and mental health across different settings can be used to interpret how time-use pattern influences the mental health of older people. This review suggests that more time-use and mental health research in older adults is required in Asian countries. The studies are essential to gather information on the daily activities of older adults from diverse communities, cultures, and various geographical locations and how this time-use could contribute to their mental health in later life. 


\subsection{Considering Robust Research Design in Time-Use and Mental Health Study}

Over half of the studies in this review applied a cross-sectional design. More longitudinal studies to examine the relationships between time-use and mental health are warranted in gerontology. Besides providing a causal relationship between independent and criterion variables, longitudinal studies are important in gerontology because it allows access changes over time and specific disease endpoints [46]. In this context, longitudinal studies provide stronger evidence on how changes in time-use could affect older people's mental health and the types of activities that older adults could participate in for better mental health. Older adults' daily life could change drastically during retirement due to work time that will be replaced by other activities and a series of psychological adjustments [47]. Therefore, it is crucial to examine time-use changes, particularly changes from pre- to post-retirement, and examine if these changes result in mental health changes. The relationship between changes in time-use and mental health should be examined in both Western and Asian countries due to cultural and lifestyle differences so that comparisons could be made $[48,49]$. By doing so, we can also understand how cultural differences in time-use could affect mental health in later life.

\subsection{Allowing Full Richness of Time-Use Data}

This review found that less than half of the studies included involved cluster analysis or latent class analytic strategy. The latent class analysis helps to simplify and integrate complex human behaviors by analyzing a number of abstract indicators that could not be directly observed [50]. The reporting of time allocation solely from time-use data is deemed to be insufficient due to the inability to make full use of the data. In time-use, latent class analysis helps capture activities as a multidimensional unit. Unique patterns that are not shown by the aggregate-level average could be discovered [51]. In other words, latent class analysis allows the full richness of time-use data to be utilized. For example, studies often assign participants to different activity levels (low, high, moderate, working, etc.) based on time-use data by cluster or latent class analysis so that the association between activity levels and mental health outcome could then be estimated. Therefore, besides reporting the average time-use for each activity, this review suggests future time-use researchers also perform cluster analysis from time-use data to identify other underlying constructs, such as activity levels (low, moderate, low, etc.) and activity patterns (regular, tedious, packed, etc.). This review also found that some studies collected time-use and mental health data but did not examine their relationships. Although there are just a few, this also reminds future studies to examine the relationship between time-use and mental health in old age to close the "critical gap" in gerontological research.

\subsection{Incorporating Demographic Issues in Time-Use and Mental Health Studies}

Demographic characteristics, particularly sex, are an important concern in gerontology. Due to different socialization in the earlier stage of life, sex differences in time-use and mental health are considered substantial issues to be considered in gerontology [52]. This is visible in Asia when men are often portrayed as the breadwinner of the family, while the women's role is highly associated with child-raising and homemaking. Past studies have shown that there are sex differences in time-use among older adults [53]. In this review, very few studies considered the sex (and other demographic variables) differences in time-use and mental health; therefore, researchers should consider examining the sex differences in time-use and if the differences affect the mental health of older adults. Aside from sex, the roles of other demographic characteristics such as marital status and work status on the relationship between time-use and mental health should be considered too.

\subsection{Limitations of This Study}

Some limitations of this scoping review are worth noting. One of the main limitations is that this review was limited to articles that were published and written in English. This may have excluded articles that were published in other languages, and therefore 
underestimating the amount of evidence. Moreover, some included studies involved a small sample size and non-random sampling technique, which will affect the findings' generalizability. Next, the present scoping review did not include studies with a qualitative design. As the time-use study also could be conducted using the observational method, and therefore underestimating those studies collected time-use data using the qualitative design. Lastly, we limited our review to studies of mental health in old age. Future reviews should also include a broad range of chronic health conditions not limited to mental health.

\section{Conclusions}

This review has provided valuable information on published studies on time-use and mental health in older people after reviewing 11 eligible studies. Although two studies did not examine the relationship between time-use and mental health, there was evidence showing that paid work, social activity, and activity diversity were linked with better mental health. Therefore, the existing evidence might support the engagement theory of aging. This review also found that older adults spent more time in passive and solitary activities during their leisure time, but successful aging is more than that as it encourages older adults to socialize with others. Findings highlight the need to continually promote an active lifestyle in old age to promote successful aging. Most importantly, this review posted several suggestions for future research on time-use and mental health: (i) although there is a substantial challenge to collect time-use data accurately across the range of daily activities, it is advisable to collect time-use data by larger time intervals (every 30-45 min) to capture the duration of each activity adequately and improve recall accuracy, (ii) other information of activity, such as with whom and where an activity was performed should also be collected to capture information on loneliness, social isolation, and social interaction, (iii) make full use of the time-use data by performing cluster analysis to generate other information, such as activity levels and patterns, (iv) measuring mental health outcome using a validated and reliable instrument, $(\mathrm{v})$ consider examining the relationship between time-use and mental health using longitudinal study design and proper statistical analysis approach, and (vi) research on time-use and mental health in older adults should be given more attention in Asia and other low- or medium-income countries.

Author Contributions: Conceptualization: S.A.H. and H.F.F.; databases search: H.F.F. and S.Y.L.; data extraction and validation: H.F.F., S.Y.L., R.K.; writing—original draft preparation: H.F.F. and S.Y.L.; writing-review and editing: S.A.H. and R.K.; supervision: S.A.H. All authors have read and agreed to the published version of the manuscript.

Funding: This work was supported by the Ministry of Education Malaysia under "Comparative Data Collection Methods for Time Use Study among Older Persons" (FRGS/1/2015/SS06/UPM/02/05).

Institutional Review Board Statement: Not applicable.

Informed Consent Statement: Not applicable.

Data Availability Statement: The data that support the findings of this study are available from the corresponding author, upon reasonable request.

Conflicts of Interest: The authors declare no conflict of interest.

\section{References}

1. Ver Ploeg, M.; Altanji, J.; Bradburn, N.; DaVanzo, J.; Nordhaus, W.; Samaniego, F. Time-Use Measurement and Research: Report of a Workshop; National Academies Press: Washington, DC, USA, 2000; ISBN 0309070929.

2. McLennan, W. How Australians Use Their Time; Australian Bureau of Statistics: Canberra, Australia, 1997.

3. Gauthier, A.H.; Smeeding, T.M. Time use at older ages: Cross-national differences. Res. Aging 2003, 25, 247-274. [CrossRef]

4. Punyakaew, A.; Lersilp, S.; Putthinoi, S. Active ageing level and time use of elderly persons in a Thai suburban community. Occup. Ther. Int. 2019, 2019, 7092695. [CrossRef]

5. Mckenna, K.; Broome, K.; Liddle, J. What older people do: Time use and exploring the link between role participation and life satisfaction in people aged 65 years and over. Aust. Occup. Ther. J. 2007, 54, 273-284. [CrossRef]

6. Kahneman, D.; Krueger, A.B.; Schkade, D.A.; Schwarz, N.; Stone, A.A. A survey method for characterizing daily life experience: The day reconstruction method. Science 2004, 306, 1776-1780. [CrossRef] 
7. Harvey, A.S. Guidelines for time use data collection and analysis. In Time Use Research in the Social Sciences; Pentland, W.E., Harvey, A.S., Lawton, M.P., McColl, M.A., Eds.; Springer: Boston, MA, USA, 2002; pp. 19-46. ISBN 978-0-306-47155-1.

8. Poblete-Valderrama, F.; Rivera, C.F.; Petermann-Rocha, F.; Leiva, A.M.; Martínez-Sanguinetti, M.A.; Troncoso, C.; Mardones, L.; Villagrán, M.; Nazar, G.; Ulloa, N.; et al. Physical activity and sedentary behaviours are associated with cognitive impairment in Chilean older adults. Rev. Med. Chile 2019, 147, 1247-1255. [CrossRef]

9. James, B.D.; Wilson, R.S.; Barnes, L.L.; Bennett, D.A. Late-life social activity and cognitive decline in old age. J. Int. Neuropsychol. Soc. 2011, 17, 998-1005. [CrossRef]

10. Won, D.; Bae, J.S.; Byun, H.; Seo, K.B. Enhancing subjective well-being through physical activity for the elderly in Korea: A meta-analysis approach. Int. J. Environ. Res. Public Health 2020, 17, 262. [CrossRef]

11. Paggi, M.E.; Jopp, D.; Hertzog, C. The Importance of Leisure Activities in the Relationship between Physical Health and Well-Being in a Life Span Sample. Gerontology 2016, 62, 450-458. [CrossRef]

12. Michèle, J.; Guillaume, M.; Alain, T.; Nathalie, B.; Claude, F.; Kamel, G. Social and leisure activity profiles and well-being among the older adults: A longitudinal study. Aging Ment. Health 2019, 23, 77-83. [CrossRef] [PubMed]

13. Lindwall, M.; Larsman, P.; Hagger, M.S. The Reciprocal Relationship Between Physical Activity and Depression in Older European Adults: A Prospective Cross-Lagged Panel Design Using SHARE Data. Health Psychol. 2011, 30, 453-462. [CrossRef] [PubMed]

14. Tardif, D. Global elderly care in crisis. Lancet 2014, 383, 927.

15. World Health Organization. Mental Mealth and Older Adults. Available online: http://www.who.int/mediacentre/factsheets/ fs381/en/ (accessed on 9 August 2017).

16. Fiske, A.; Wetherell, J.L.; Gatz, M. Depression in Older Adults. Annu. Rev. Clin. Psychol. 2009, 5, 363-389. [CrossRef] [PubMed]

17. De Mendonça Lima, C.A.; Ivbijaro, G. Mental health and wellbeing of older people: Opportunities and challenges. Ment. Health Fam. Med. 2013, 10, 125-127.

18. Guven, C.; Saloumidis, R. Life Satisfaction and Longevity: Longitudinal Evidence from the German Socio-Economic Panel. Ger. Econ. Rev. 2014, 15, 453-472. [CrossRef]

19. Strawbridge, W.J.; Deleger, S.; Roberts, R.E.; Kaplan, G.A. Physical activity reduces the risk of subsequent depression for older adults. Am. J. Epidemiol. 2002, 156, 328-334. [CrossRef]

20. Dinas, P.C.; Koutedakis, Y.; Flouris, A.D. Effects of exercise and physical activity on depression. Ir. J. Med. Sci. 2011, 180, 319-325. [CrossRef] [PubMed]

21. Kim, J.; Lee, S.; Chun, S.; Han, A.; Heo, J. The effects of leisure-time physical activity for optimism, life satisfaction, psychological well-being, and positive affect among older adults with loneliness. Ann. Leis. Res. 2017, 20, 406-415. [CrossRef]

22. Randall, G.K.; Martin, P.; Johnson, M.A.; Poon, L.W. Successful aging: A psychosocial resources model for very old adults. J. Aging Res. 2012, 2012, 934649. [CrossRef]

23. Johnson, J.K.M.; Sarkisian, N.; Williamson, J.B. Using a micro-level model to generate a macro-level model of productive successful aging. Gerontologist 2015, 55, 107-119. [CrossRef]

24. Kleineidam, L.; Thoma, M.V.; Maercker, A.; Bickel, H.; Mösch, E.; Hajek, A.; König, H.H.; Eisele, M.; Mallon, T.; Luck, T.; et al. What Is Successful Aging? A Psychometric Validation Study of Different Construct Definitions. Gerontologist 2019, 59, 738-748. [CrossRef]

25. Rowe, J.W.; Kahn, R.L. Successful Aging. Gerontologist 1997, 37, 433. [CrossRef]

26. Carr, K.; Weir, P. A Conceptual Model of Engagement Profiles Throughout the Decades of Older Adulthood. Front. Psychol. 2019, 10, 2535. [CrossRef]

27. Morrow-Howell, N.; Greenfield, E.A. Productive engagement in later life. In Handbook of Aging and the Social Sciences, 8th ed.; Academic Press: Cambridge, MA, USA, 2016; ISBN 9780124172357.

28. Arksey, H.; O’Malley, L. Scoping studies: Towards a methodological framework. Int. J. Soc. Res. Methodol. 2005, 8, 19-32. [CrossRef]

29. Richardson, W.S.; Wilson, M.C.; Nishikawa, J.; Hayward, R.S. The well-built clinical question: A key to evidence-based decisions. ACP J. Club 1995, 123, A12-A13. [CrossRef]

30. Colquhoun, H.L.; Levac, D.; O’Brien, K.K.; Straus, S.; Tricco, A.C.; Perrier, L.; Kastner, M.; Moher, D. Scoping reviews: Time for clarity in definition, methods, and reporting. J. Clin. Epidemiol. 2014, 67, 1291-1294. [CrossRef] [PubMed]

31. Prigerson, H.G.; Monk, T.H.; Reynolds, C.F.; Begley, A.; Houck, P.R.; Bierhals, A.J.; Kupfer, D.J. Lifestyle regularity and activity level as protective factors against bereavement-related depression in late-life. Depression 1995, 3, 297-302. [CrossRef]

32. Jennings, J.M.; Darwin, A.L. Efficacy Beliefs, Everyday Behavior, and Memory Performance Among Older Elderly Adults. Educ. Gerontol. 2003, 29, 71-91. [CrossRef]

33. Hahn, E.A.; Cichy, K.E.; Almeida, D.M.; Haley, W.E. Time use and well-being in older widows: Adaptation and resilience. J. Women Aging 2011, 23, 149-159. [CrossRef] [PubMed]

34. Liddle, J.; Gustafsson, L.; Bartlett, H.; Mckenna, K. Time use, role participation and life satisfaction of older people: Impact of driving status. Aust. Occup. Ther. J. 2012, 59, 384-392. [CrossRef] [PubMed]

35. Tadic, M.; Oerlemans, W.G.M.; Bakker, A.B.; Veenhoven, R. Daily Activities and Happiness in Later Life: The Role of Work Status. J. Happiness Stud. 2013, 14, 1507-1527. [CrossRef]

36. Morrow-Howell, N.; Putnam, M.; Lee, Y.S.; Greenfield, J.C.; Inoue, M.; Chen, H. An investigation of activity profiles of older adults. J. Gerontol. Ser. B Psychol. Sci. Soc. Sci. 2014, 69, 809-821. [CrossRef] [PubMed] 
37. Adjei, N.K.; Jonsson, K.R.; Brand, T. Time spent on work-related activities, social activities and time pressure as intermediary determinants of health disparities among elderly women and men in 5 European countries: A structural equation model. Int. J. Equity Health 2018, 17, 121. [CrossRef]

38. Lee, S.; Koffer, R.E.; Sprague, B.N.; Charles, S.T.; Ram, N.; Almeida, D.M. Activity diversity and its associations with psychological well-being across adulthood. J. Gerontol. Ser. B Psychol. Sci. Soc. Sci. 2018, 73, 985-995. [CrossRef]

39. Olds, T.; Burton, N.W.; Sprod, J.; Maher, C.; Ferrar, K.; Brown, W.J.; Van Uffelen, J.; Dumuid, D. One day you'll wake up and won't have to go to work: The impact of changes in time use on mental health following retirement. PLoS ONE 2018, 13, e0199605. [CrossRef]

40. Chen, Y.C.; Putnam, M.; Lee, Y.S.; Morrow-Howell, N. Activity Patterns and Health Outcomes in Later Life: The Role of Nature of Engagement. Gerontologist 2019, 59, 698-708. [CrossRef]

41. Bauman, A.; Bittman, M.; Gershuny, J. A short history of time use research; Implications for public health. BMC Public Health 2019, 19, 607. [CrossRef] [PubMed]

42. Gopalkrishnan, N. Cultural Diversity and Mental Health: Considerations for Policy and Practice. Front. Public Health 2018, 6, 179. [CrossRef]

43. Zhang, J.; Emery, T.; Dykstra, P. Grandparenthood in China and Western Europe: An analysis of CHARLS and SHARE. Adv. Life Course Res. 2020, 45, 100257. [CrossRef]

44. Schwingel, A.; Niti, M.M.; Tang, C.; Ng, T.P. Continued work employment and volunteerism and mental well-being of older adults: Singapore longitudinal ageing studies. Age Ageing 2009, 38, 531-537. [CrossRef] [PubMed]

45. Fernández-Niño, J.A.; Bonilla-Tinoco, L.J.; Manrique-Espinoza, B.S.; Romero-Martínez, M.; Sosa-Ortiz, A.L. Work status, retirement, and depression in older adults: An analysis of six countries based on the Study on Global Ageing and Adult Health (SAGE). SSM Popul. Health 2018, 6, 1-8. [CrossRef] [PubMed]

46. Newman, A.B. An Overview of the Design, Implementation, and Analyses of Longitudinal Studies on Aging. J. Am. Geriatr. Soc. 2010, 58, S287-S291. [CrossRef]

47. Robinson, O.C.; Demetre, J.D.; Corney, R.H. The variable experiences of becoming retired and seeking retirement guidance: A qualitative thematic analysis. Br. J. Guid. Couns. 2011, 39, 239-258. [CrossRef]

48. Luborsky, M.R.; LeBlanc, I.M. Cross-cultural perspectives on the concept of retirement: An analytic redefinition. J. Cross Cult. Gerontol. 2003, 18, 251-271. [CrossRef] [PubMed]

49. Aw, S.; Koh, G.; Oh, Y.J.; Wong, M.L.; Vrijhoef, H.J.M.; Harding, S.C.; Geronimo, M.A.B.; Lai, C.Y.F.; Hildon, Z.J.L. Explaining the continuum of social participation among older adults in Singapore: From 'closed doors' to active ageing in multi-ethnic community settings. J. Aging Stud. 2017, 42, 46-55. [CrossRef]

50. Fairley, L.; Cabieses, B.; Small, N.; Petherick, E.S.; Lawlor, D.A.; Pickett, K.E.; Wright, J. Using latent class analysis to develop a model of the relationship between socioeconomic position and ethnicity: Cross-sectional analyses from a multi-ethnic birth cohort study. BMC Public Health 2014, 14, 835. [CrossRef]

51. Hunt, E.; McKay, E.A. A scoping review of time-use research in occupational therapy and occupational science. Scand. J. Occup. Ther. 2015, 22, 1-12. [CrossRef]

52. Sinnott, J.D.; Shifren, K. Gender and aging: Gender differences and gender roles. In Handbook of the Psychology of Aging; Birren, J.E., Schaie, K.W., Eds.; Academic Press: San Diego, CA, USA, 2001; pp. 454-476.

53. Gauthier, A.H.; Smeeding, T.M. Historical trends in the patterns of time use of older adults. In Aging in Advanced Industrial States; Springer: Dordrecht, The Netherlands, 2010. 